\title{
Multiple Spin State Analysis of Magnetic Nano Graphene
}

\author{
Norio Ota, Narjes Gorjizadeh* and Yoshiyuki Kawazoe* \\ Pure and Applied Sciences, University of Tsukuba, 1-1-1 Tenoudai, Tsukuba-city, Ibaraki 305-8571 JAPAN \\ *Institute for Materials Research, Tohoku University., 2-1-1 Katahira, Aoba-ku, Sendai 980-8577 JAPAN
}

Recent experiments have suggested a possibility of room-temperature ferromagnetism in graphite-like materials. We analyzed multiple spin states in asymmetric graphene molecules to find the mechanism responsible for ferromagnetism. First principle density functional theory was applied to calculate ground state spin density, energy, and atom position depending on each spin state. Molecules with dihydrogenated zigzag edges like $\mathrm{C}_{64} \mathrm{H}_{27}$, $\mathrm{C}_{56} \mathrm{H}_{24}, \mathrm{C}_{64} \mathrm{H}_{25}, \mathrm{C}_{56} \mathrm{H}_{22}$, and $\mathrm{C}_{64} \mathrm{H}_{23}$ indicated that the highest spin state in every molecule is the most stable having an energy difference of $\mathrm{kT}=3000 \mathrm{~K}$ with the next spin state. In contrast, nitrogen substituted molecules like $\mathrm{C}_{59} \mathrm{~N}_{5} \mathrm{H}_{22}, \mathrm{C}_{52} \mathrm{~N}_{4} \mathrm{H}_{20}, \mathrm{C}_{61} \mathrm{~N}_{3} \mathrm{H}_{22}, \mathrm{C}_{54} \mathrm{~N}_{2} \mathrm{H}_{20}$, and $\mathrm{C}_{63} \mathrm{~N}_{1} \mathrm{H}_{22}$ demonstrated opposite results where the lowest spin state was the most stable. The magnetic stability of graphene molecules can be explained through three key factors depending on the edge specified localized spin state, the exchange interaction between parallel spins inside a molecule, and optimized atom position. We intend to apply these results to design carbon-based magnets, ultra high density information storage, and spintronic devices.

Key words: graphene, ferromagnetism, carbon material, first principle calculation

\section{Introduction}

Carbon based room-temperature ferromagnetic materials are very attractive in many applications such as a light weight ecological magnet ${ }^{1)-4)}$, an ultra high density 100 tera bit/inch ${ }^{2}$ class information storage ${ }^{5)}$ and novel spintronic devices ${ }^{6)-7)}$. Recently several experiments have suggested a possibility of room-temperature ferromagnetism in modified graphite materials. Esquinazi et al. ${ }^{8)}$ predicted ferromagnetic magnetization loop in proton ion irradiated graphite. Magnetic moments with orders of $5 \times 10^{-6} \mathrm{emu}$ were observed at a room-temperature having a coercive force of 100 Oe. Kamishima et al. ${ }^{9}$ synthesized magnetically attracted graphite-like powder. Estimated Curie temperature was very high, up to $800 \mathrm{~K}$, having 0.5 emu/g saturation magnetization. Recent experiment by Wang et al ${ }^{10)}$ indicated that reduced graphene oxide shows $0.02 \mathrm{emu} / \mathrm{g}$ saturation magnetization at $300 \mathrm{~K}$. Also, strong magnetism was observed at the defect edge of cracked graphite by Cervenka et al. ${ }^{11)}$. Those experiments encouraged us to open a new door to carbon ferromagnetism.

There were many theoretical predictions on zigzag edge carbon magnetism due to localized density of states near Fermi energy ${ }^{12)-16)}$ resulting antiferromagnetic feature with total magnetization of zero. Also, rectangular shaped graphene nano dot has been extensively studied ${ }^{17)-20)}$, but only shows singlet state with zero magnetization. Whereas, Kusakabe and Maruyama ${ }^{21) 22)}$ proposed an asymmetric ribbon model showing ferrimagnetic behavior with non-zero total magnetization. This gave us a hint to analyze basic magnetism of graphene and graphite based materials. Unfortunately, they did not apply to nano-meter-length graphene, and did not analyze multiple spin states.

Reported experiments mostly use powder samples, which suggested us to apply to molecules. In such molecule, there appears complex multiple spin states. Questions are, which is the most stable total molecular energy state and how about energy difference between spin states. In a previous paper ${ }^{23)}$, we proposed $\mathrm{C}_{48} \mathrm{H}_{28}$ molecule model with dihydrogenated zigzag edge. The first principle theory calculation shows that high spin state $(\mathrm{Sz}=3 / 2)$ is more stable than low spin state $(\mathrm{Sz}=1 / 2)$. Such results taught us the importance of multiple spin state analysis in systematic molecule group. This paper reports multiple spin state analysis in two groups. One is dihydrogenated zigzag edge molecules like $\mathrm{C}_{64} \mathrm{H}_{27}, \mathrm{C}_{56} \mathrm{H}_{24}, \mathrm{C}_{64} \mathrm{H}_{25}, \mathrm{C}_{56} \mathrm{H}_{22}$ and $\mathrm{C}_{64} \mathrm{H}_{23}$. The other is nitrogen substituted molecules like $\mathrm{C}_{59} \mathrm{~N}_{5} \mathrm{H}_{22}, \mathrm{C}_{52} \mathrm{~N}_{4} \mathrm{H}_{20}, \mathrm{C}_{61} \mathrm{~N}_{3} \mathrm{H}_{22}, \mathrm{C}_{54} \mathrm{~N}_{2} \mathrm{H}_{20}$ and $\mathrm{C}_{63} \mathrm{~N}_{1} \mathrm{H}_{22}$ Through those analysis, we could find that high spin state of dihydrogenated molecules are stable than lower one. 


\section{2, Application Example}

\section{and Selections of Calculation Model}

One of the important applications of nano-meter-size ferromagnetic materials is information storage. Current hard disc storage has a density around 0.7 tera-bit/inch ${ }^{2}$ and now targeting 1 tera-bit /inch ${ }^{2}$ density 5) with $10 \mathrm{~nm}$ length, $25 \mathrm{~nm}$ width magnetic mark. Whereas, recent scientific report have predicted a possibility of single atom base storage 24 ) with density of $10^{4}$ tera-bit/inch ${ }^{2}$. There is a missing link between those two density regions. In order to link those two regions, one promising candidate is a ferromagnetic molecule dot array having a typical unit size of $1 \mathrm{~nm}$ length, $2.5 \mathrm{~nm}$ width having $10^{2}$ tera-bit /inch $^{2}$. Fig.1 shows a magnetic recording image using such molecule array.

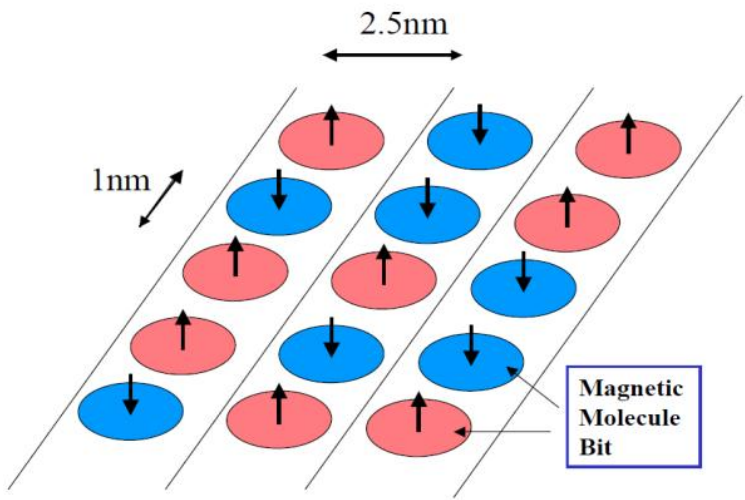

Fig.1 Ultra-high density 100 tera-bit/inch ${ }^{2}$ magnetic recording image using ferromagnetic molecule dot array.

For modeling such nano-meter-size molecule having asymmetric dihydrogenated zigzag edges, our calculation tried five typical examples like $\mathrm{C}_{64} \mathrm{H}_{27}$, $\mathrm{C}_{64} \mathrm{H}_{25}, \mathrm{C}_{64} \mathrm{H}_{23}, \mathrm{C}_{56} \mathrm{H}_{24}$ and $\mathrm{C}_{56} \mathrm{H}_{22}$ as illustrated in Fig.2. Those molecules have different numbers of dihydrogenated edges $\left(\mathrm{CH}_{2}\right.$-edge $)$ from five to one. Molecule size of $\mathrm{C}_{64} \mathrm{H}_{27}$ is $2.16 \mathrm{~nm} \times 0.93 \mathrm{~nm}$.

\section{Calculation Methods}

In order to clarify magnetism, we obtained spin density, total molecular energy, and optimized atom arrangement. Density functional theory (DFT) 25)26) based generalized gradient approximation method (GGA-UPBEPBE) ${ }^{27}$ ) is applied for those calculations. Functional basis is $6-31 \mathrm{G}{ }^{28}$ ). For obtaining accurate self consistent field energy, requested convergence on root mean square density matrix was less than 10E-8 within 128 cycles.

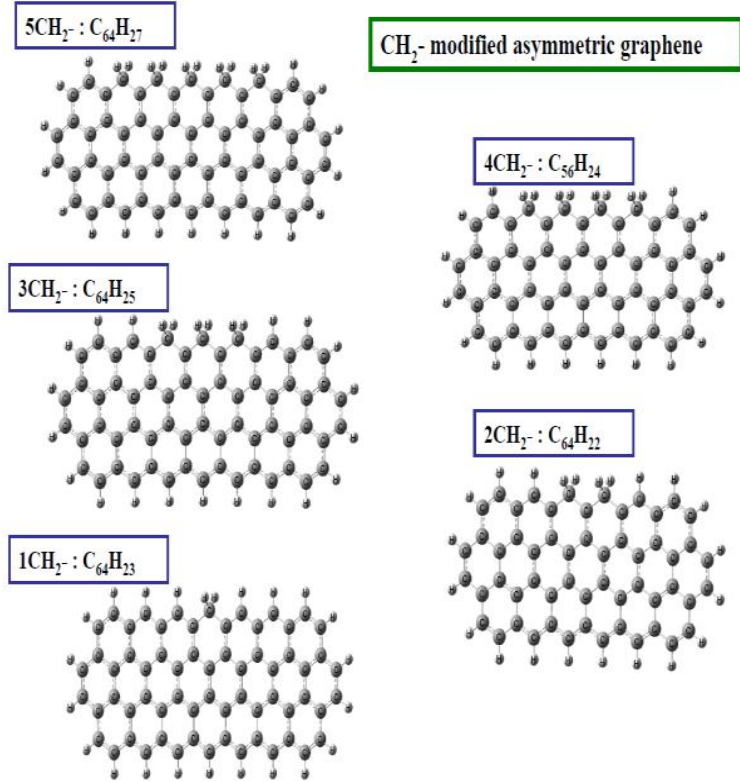

Fig.2 Five model molecules with different numbers of dihydrogenated zigzag edges: $\mathrm{C}_{64} \mathrm{H}_{27}, \mathrm{C}_{56} \mathrm{H}_{24}, \mathrm{C}_{64} \mathrm{H}_{25}$, $\mathrm{C}_{56} \mathrm{H}_{22}$, and $\mathrm{C}_{64} \mathrm{H}_{23}$. Large balls are carbon atoms, and small ones are hydrogen.

\section{4, Dihydrogenated Graphene-like Molecule}

In $\mathrm{C}_{64} \mathrm{H}_{27}$ molecule with five $\mathrm{CH}_{2}$-modified edges, there are five unpaired electrons in a whole molecule. In such a case, maximum possible total molecular spin $\mathrm{Sz}$ is $5 / 2$ (the highest spin state), next one is $3 / 2$, and minimum one is $1 / 2$ (the lowest spin state). By optimizing both atom position and molecular energy by DFT calculation, we could obtained spin density maps at a contour surface of $0.001 \mathrm{e}^{-} \mathrm{A}^{3}$ as shown in Fig.3, where up spin is shown in red (dark gray), down spin in blue (light gray). Looking at those figures, we can notice the following features,

(1) In every spin state, localized spin density at $\mathrm{CH}_{2}$-edge shows similar feature, that is, there appears up spin on two hydrogen sites and down spin on zigzag edge carbon. Localized ( $\mathrm{CH}_{2}$-orbit) molecular Hund-rule plays a major role for such edge specified spin density distribution. In unlimited length ribbon case, this causes zigzag edge ferri-magnetism ${ }^{12) 21)}$. Advantage of molecule model is a real space representation for easier understanding of magnetism.

(2) In case of $\mathrm{S}_{z}=5 / 2$, inside a molecule, up and down spins are alternately arranged one by one very regularly.

(3) Whereas, in case of $\mathrm{Sz}=3 / 2$ and $1 / 2$, there appears up-up and down-down spin pairs inside a molecule. 
Exchange coupling between up-up (down-down) spins makes the local binding energy increase and finally elevates total molecular energy. In $\mathrm{C}_{64} \mathrm{H}_{27}$ molecule, total molecular energy is calculated and compared with each other. Results are shown in Fig.4. The lowest energy is obtained in the spin state $\mathrm{Sz}=5 / 2$. Energy difference between $\mathrm{Sz}=5 / 2$ and $\mathrm{Sz}=3 / 2$ is $6.1 \mathrm{kcal} / \mathrm{mol}$.
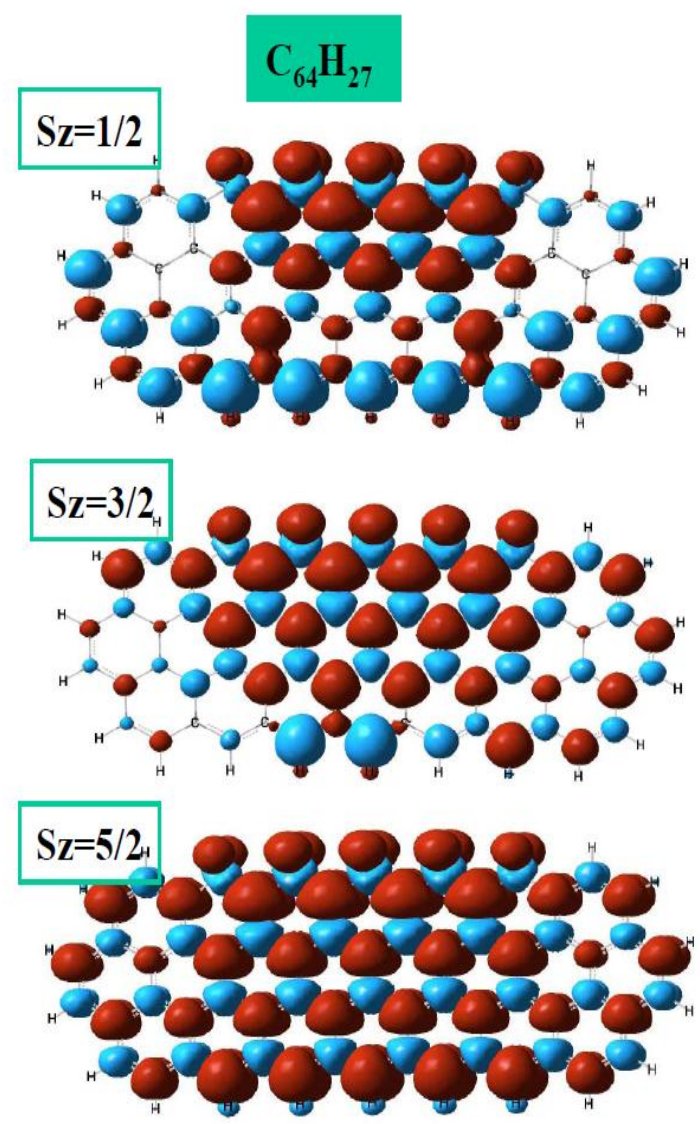

Fig.3 Spin density map of $\mathrm{C}_{64} \mathrm{H}_{27}$ molecule having three spin states of $\mathrm{Sz}=1 / 2,3 / 2$, and $5 / 2$. Red (dark gray) shows up-spin, whereas blue (light gray) indicates down-spin on $0.001 \mathrm{e}^{-} / \mathrm{A}^{3}$ spin-density-contour surface. This view is slightly left-hand side tilted.

In order to obtain more systematic behavior, we changed numbers of $\mathrm{CH}_{2}$ - modified edges, as $\mathrm{C}_{56} \mathrm{H}_{24}$ (four $\mathrm{CH}_{2}$ - edges), $\mathrm{C}_{64} \mathrm{H}_{25}$ (three), $\mathrm{C}_{56} \mathrm{H}_{22}$ (two) and $\mathrm{C}_{64} \mathrm{H}_{23}$ (one). Every molecule has several spin states depending on numbers of unpaired electrons in a molecule. Energy differences between spin states are calculated and results are shown in Fig. 4. It is very clear that in every molecule the highest spin state is the lowest and stable energy state under the condition of ground state DFT calculation comparison.

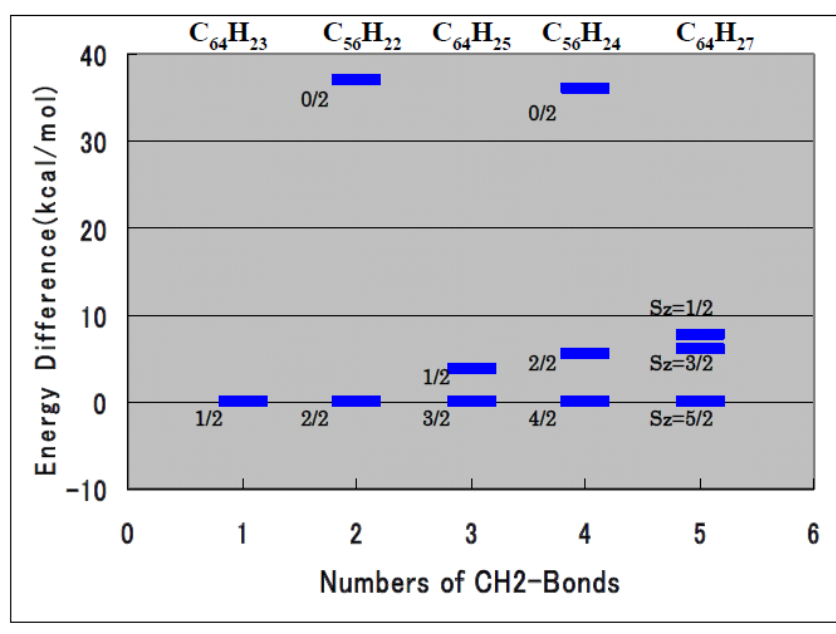

Fig.4 Energy difference between spin states for five $\mathrm{CH}_{2}-$ modified molecules. The highest spin state is the most stable one in every molecule.

\section{5, Nitrogen Substituted Graphene Molecule}

Triggered by an experiment of K. Kamishima et $\mathrm{al}^{4)}$, which uses nitrogen contained small molecule as a starting material, we imagined some nitrogen atoms may substitute zigzag edge carbons and may bring strong magnetism. Model molecules are shown in Fig.5, where nitrogen atoms are illustrated by blue balls.

In $\mathrm{C}_{59} \mathrm{~N}_{5} \mathrm{H}_{22}$ molecule, there are five unpaired electrons, which enable three spin states as $\mathrm{Sz}=1 / 2,3 / 2$ and 5/2. Spin density map is calculated as shown in Fig.6. Features are summarized as follows,

(1) In case of $\mathrm{Sz}=1 / 2$, there occurs canceling of spins around nitrogen atoms (blue $\mathrm{N}$ marks), also there appears up and down alternative spin array around opposite side zigzag edge.

(2) Whereas in $\mathrm{Sz}=3 / 2$, many up-up spin pairs appear on every $\mathrm{NH}^{-}$site. Also in $\mathrm{Sz}=5 / 2$, stronger up-up spin pairs appear. These many parallel spin pairs may increase exchange interaction and finally bring unfavorable molecular energy increase

Above observation suggests the lowest and stable energy to be $\mathrm{Sz}=1 / 2$ or $0 / 2$ in $\mathrm{NH}^{-}$edge graphene molecule.

Also, we found that optimized atomic position drastically changes depending on spin state as shown in Fig.7. Right illustrations in Fig.7 indicate tilt angle $\theta$ of hydrogen atom from a molecular plane.

(1)In case of $\mathrm{Sz}=1 / 2$, four $\mathrm{NH}^{-}$site show $\mathrm{SP}^{2}$-like atomic arrangements which brings almost no spin density around top zigzag edge. Only at center 
$\mathrm{NH}$-site, we can see $\mathrm{SP}^{3}$ like atomic arrangement, which may bring up and down spin alternative arrangements on opposite zigzag edge.

Concerning $\mathrm{SP}^{2}$ positioned nitrogen, bond length between nitrogen and neighbor carbon is $0.1412 \mathrm{~nm}$, which is $0.7 \%$ shorter than carbon to carbon length of $0.1421 \mathrm{~nm}$. Angle of C-N-C is 119 degree, whereas $\mathrm{C}-\mathrm{C}-\mathrm{C}$ is 122 degree. Such slight atomic position change gives remarkable spin density change at a zigzag edge corner.

(2)In case of $\mathrm{Sz}=3 / 2$ and 5/2, all $\mathrm{NH}^{-}$sites show $\mathrm{SP}^{3}$-like arrangements at zigzag edge. Such arrangement brings strong exchange coupling between up spin on hydrogen and up spin on nitrogen.

(3)We checked $\mathrm{CH}_{2}$-modified molecule case. In all molecules and every spin state we can not find any drastic change in atomic arrangements.

We added calculations on four molecules as like $\mathrm{C}_{52} \mathrm{~N}_{4} \mathrm{H}_{20}$ (four $\mathrm{NH}^{-}$edges), $\mathrm{C}_{61} \mathrm{~N}_{3} \mathrm{H}_{22}$ (three), $\mathrm{C}_{54} \mathrm{~N}_{2} \mathrm{H}_{20}$ (two) and $\mathrm{C}_{63} \mathrm{~N}_{1} \mathrm{H}_{22}$ (one).

As shown in Fig.8, energy difference between spin states is completely opposite with that of $\mathrm{CH}_{2}$-cases. In every molecule, lowest energy and stable spin state is the lowest spin state to be $\mathrm{Sz}=1 / 2$ or $0 / 2$. We cannot expect strong magnetism by such nitrogen substituted asymmetric molecules
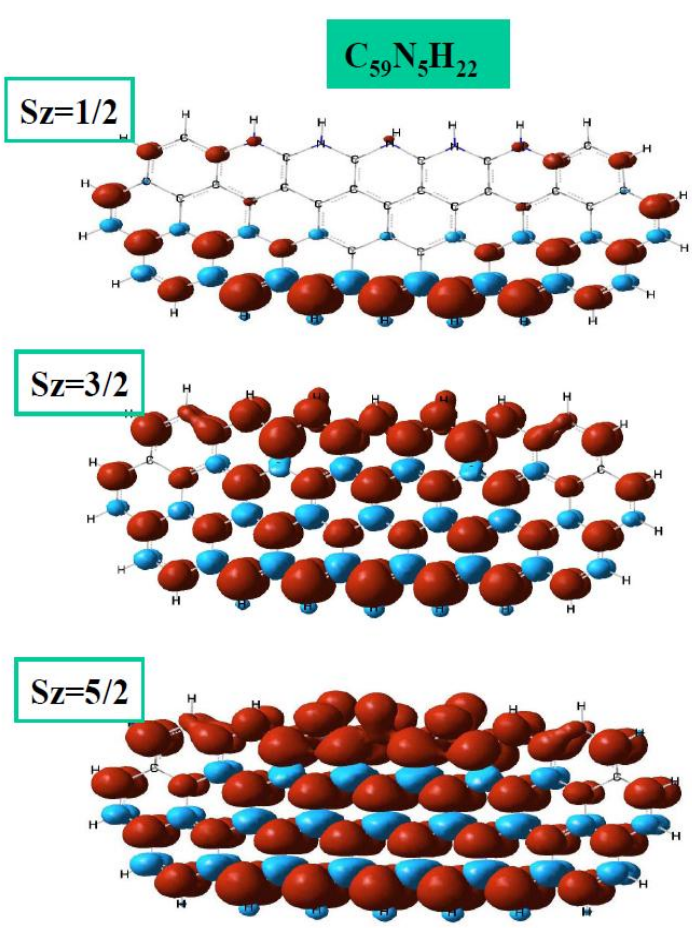

Fig. 6. Spin density map of $\mathrm{C}_{59} \mathrm{~N}_{5} \mathrm{H}_{22}$ molecule having three spin states of $\mathrm{Sz}=1 / 2,3 / 2$, and $5 / 2$ at a contour surface of $0.001 \mathrm{e} / \mathrm{A}^{3}$.

\section{$\mathrm{C}_{59} \mathrm{~N}_{5} \mathrm{H}_{22}$}
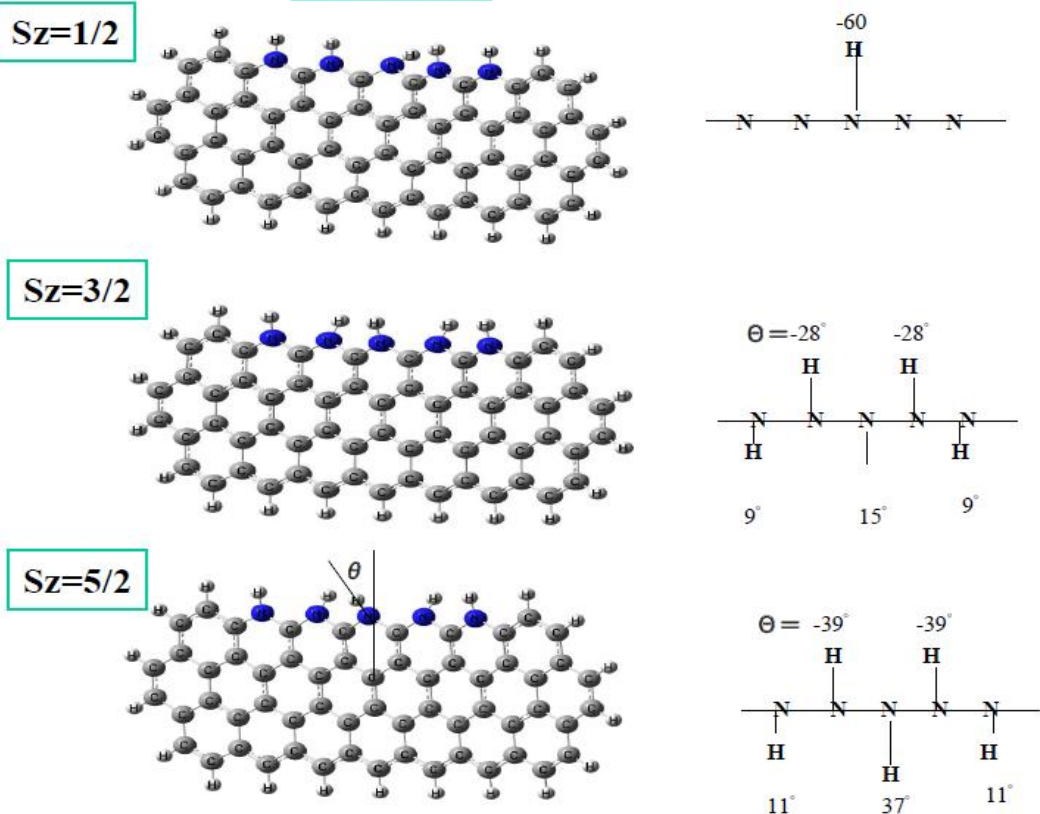

Fig.7 Atoms in $\mathrm{C}_{59} \mathrm{~N}_{5} \mathrm{H}_{22}$ molecule have different arrangements depending on spin states. Three figures at left are plane views observed slightly tilting from right. Illustrations at right indicate tilt angle $\theta$ of hydrogen atom bonded to nitrogen from top view molecules. Blue balls indicate nitrogen atoms.
Fig.5 Nitrogen-substituted graphene

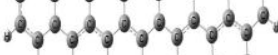

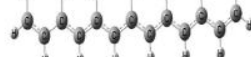

s.

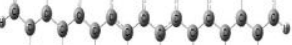

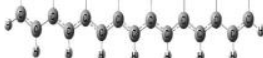

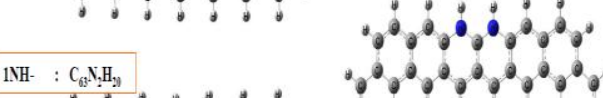

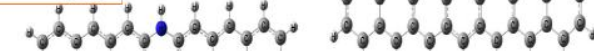

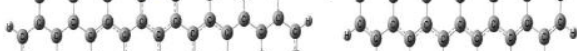

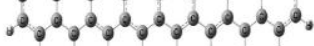

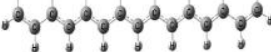

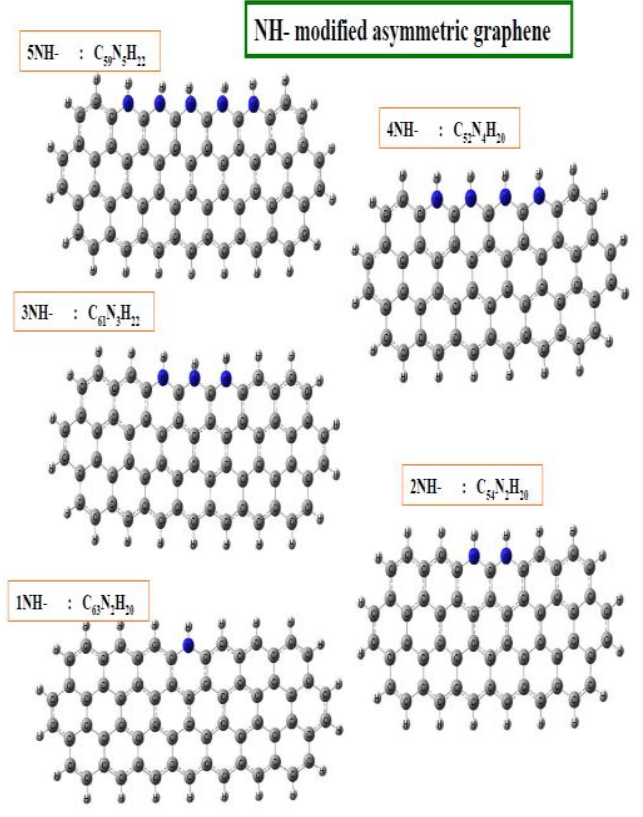


tera bit/inch ${ }^{2}$ class information storage and novel spintronic devices.

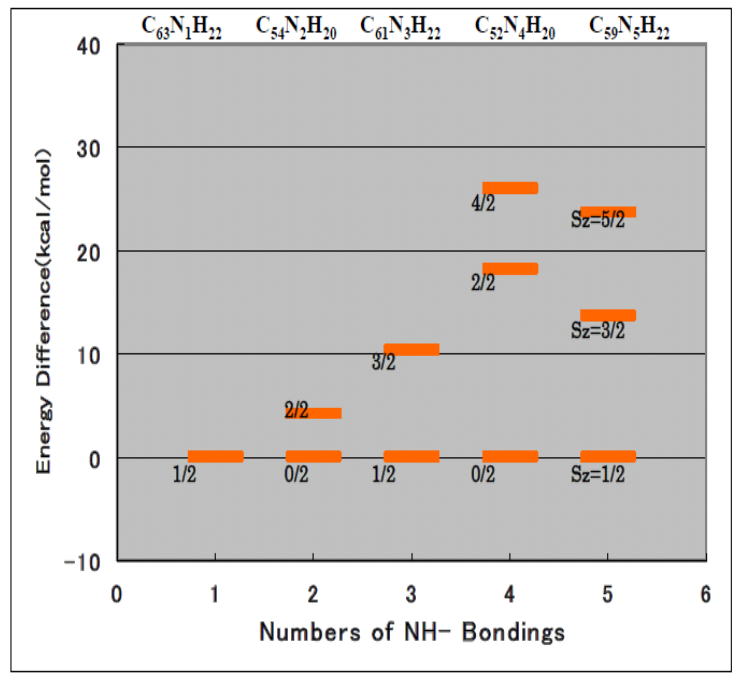

Fig. 8 Energy difference between spin states for five NH-modified molecules. Lowest spin state show most stable in every molecule.

\section{5 , Conclusion}

Recently, several experiments on graphite like materials have suggested a possibility of room-temperature ferromagnetism. In order to find out such mechanism, multiple spin state analysis is applied to nano-meter-size graphene-like molecules. First principle density functional theory is applied to calculate spin density distribution, molecular energy and optimized atomic position depending on each spin state.

(1) Molecules with dihydrogenated $\left(\mathrm{CH}_{2}{ }^{-}\right)$zigzag edges like $\mathrm{C}_{64} \mathrm{H}_{27}, \mathrm{C}_{56} \mathrm{H}_{24}, \mathrm{C}_{64} \mathrm{H}_{25}, \mathrm{C}_{56} \mathrm{H}_{22}$ and $\mathrm{C}_{64} \mathrm{H}_{23}$ show that in every molecule the highest spin state is the most stable. Energy difference between the most stable spin state and the next one overcomes $6 \mathrm{kcal} / \mathrm{mol}$ which is $\mathrm{kT}=3000 \mathrm{~K}$ in temperature representation.

(2) In contrast, nitrogen substituted asymmetric molecules like $\mathrm{C}_{59} \mathrm{~N}_{5} \mathrm{H}_{22}, \mathrm{C}_{52} \mathrm{~N}_{4} \mathrm{H}_{20}, \mathrm{C}_{61} \mathrm{~N}_{3} \mathrm{H}_{22}, \mathrm{C}_{54} \mathrm{~N}_{2} \mathrm{H}_{20}$ and $\mathrm{C}_{63} \mathrm{~N}_{1} \mathrm{H}_{22}$ show the opposite result, that is, in every molecule the lowest spin state is the most stable as $\mathrm{Sz}=1 / 2$ or $0 / 2$. We cannot explain strong magnetism by such nitrogen substituted zigzag edge molecule model.

(3) Magnetic stability of graphene-like molecule can be explained by following three key issues,

1) Zigzag edge specified localized spin density,

2) Exchange interactions between parallel spins (up-up or down-down) inside of a molecule,

3) Atom position optimization depending on spin state.

Those results are useful to design a future carbon-base ferro-magnet, an ultra high density 100

\section{Acknowledgements}

Narjes Gorjizadeh would like to thank the crew of the Center for Computational Materials Science, Institute for Materials Research of Tohoku University for their support of the Hitachi SR11000(model K2) supercomputer system, and Global COE Program "Materials Integration (International Center of Education and Research),Tohoku University," MEXT, Japan, for financial support.

\section{References}

1) A. A. Ovchinnikov and V. N. Spector: Synth. Met., 27, B615 (1988)

2) K. Murata, H. Ueda and K. Kawaguchi: Synth. Met., 44, 357 (1991)

3) K. Murata, H. Ushijima, H. Ueda and K. Kawaguchi: J. Chem. Soc., Chem. Commun., 7,567 (1992)

4) J. S. Miller: Inorg.Chem.39, 4392 (2000)

5) Y. Shiroishi, F. Fukuda, I. Tagawa, H. Iwasaki, S. Takenoiri, H. Tanaka, M. Mutoh and N. Yoshikawa : IEEE Trans. On Magnetics, 45, 3816 (2009)

Y. Shiroishi: Magune, 5, (2010), Review in Japanese

6) M. Ohishi, M. Shiraishi, R. Nouchi, T. Nozaki, T. Shinjyo and Y. Suzuki: Jpn. J. Appl. Phys. 46, L605 (2007) Seiji Shiraishi: Surface Science、29、310 (2008), Review in Japanese

7) N. Tombros, C. Jozsa, M. Popinciuc, H. T. Jonkman and B. J.van Wees : Nature 448, 571 (2007)

8) P. Esquinazi, D. Spemann, R. Hohne, A. Setzer, K. Han, and T. Butz: Phys.Rev.Lett.,91, 227201 (2003)

9) K. Kamishima, T. Noda, F. Kadonome, K. Kakizaki and N. Hiratsuka: J. of Magnetism and Magnetic Materials $\mathbf{3 1 0}$, e346 (2007)

10) Y. Wang, Y. Huang, Y. Song, X. Zhang, Y. Ma, J. Liang and Y. Chen : Nano Letters ,9, (2009)220

11) J. Cervenka, M. Katsnelson and C. Flips: Nature Physics online Oct.04 (2009)

12) M. Fujita, K. Wakabayashi, K. Nakada and K. Kusakabe: J. of the Phys. Soc. of Japan .65, 1920 (1996)

13) K. Nakada, M. Fujita, G. Dresselhaus and M. Dresselhaus: Phy. Rev. B, 54, 17954 (1996)

14) K. Wakabayashi, M. Fujita, H. Ajiki and M Sigrist: Phys. Rev. B, 59, 8271 (1999)

15) Y. Miyamoto, K. Nakada and M. Fujita : Phys. Rev. B 59, 9858 (1999)

16) J. Berashevich and T. Chakraborty: Phys. Rev. B, 80, 115430 (2009)

17) H. Zheng and W. Duley: Phys. Rev. B, 78, 045421 (2008)

18) D. E. Jiang, B. G. Sumpter and S. Dai: J. Chem. Phys. 127, 124703 (2007)

19) X. Gao, Z. Zhuo, Y. Zhao, S. Nagase, S. B. Zhang and Z. 
Chen : J. Phys. Chem., C112, 12677 (2008)

20) T. Wassman, A. P. Seitsonen, A. M. Saitta, M. Lazzeri and F. Mauri: Phys. Rev. Lett., 101, 096402 (2008)

21) K. Kusakabe and M. Maruyama: Phys. Rev. B 67, 092406 (2003)

22) M. Maruyama and K. Kusakabe : J. of the Phys. Soc. of Japan , 73, .656 (2004)

23) N. Ota, N. Gorjizadeh and Y. Kawazoe: J. Magn. Soc. Jpn., 34, 573 (2010)

24) C.F. Hirjibehedin, C-Y. Lin, A.F. Otte, M. Ternes, C. P. Lutz, B. A. Jones and A. J. Heinrich: Science, 317, 1199 (2007)

25) P. Hohenberg and W. Kohn: Phys. Rev., 136, B867 (1964)

26) W.Kohn and L. Sham : Phys. Rev., 140, A1133 (1965)

27) J. P. Perdew, K. Burk and M. Ernzerhof: Phy. Rev. Lett., 77, 3865 (1966)

28) R. Ditchfield, W. Hehre and J. Pople : J. Chem. Phys. 54, 724 (1971)

Received Sep. 21 2010; Accepted May 16, 2011 